\title{
Bone-anchored hearing system, contralateral routing of signals hearing aid or cochlear implant: what is best in single-sided deafness?
}

\author{
Till F. Jakob ${ }^{1} \mathbb{D}$ - Iva Speck ${ }^{1}$ Ann-Kathrin Rauch ${ }^{1} \cdot$ Frederike Hassepass $^{1} \cdot$ Manuel C. Ketterer $^{1} \cdot$ Rainer Beck $^{1}$. \\ Antje Aschendorff ${ }^{1} \cdot$ Thomas Wesarg $^{1} \cdot$ Susan Arndt ${ }^{1}$
}

Received: 4 November 2020 / Accepted: 20 January 2021 / Published online: 10 February 2021

(c) The Author(s) 2021

\begin{abstract}
Purpose The aim of the study was to compare long-term results after 1 year in patients with single-sided deafness (SSD) who were fitted with different hearing aids. The participants tested contralateral routing of signals (CROS) hearing aids and bone-anchored hearing systems (BAHS). They were also informed about the possibility of a cochlear implant (CI) and chose one of the three devices. We also investigated which factors influenced the choice of device.

Methods Prospective study with 89 SSD participants who were divided into three groups by choosing BAHS, CROS, or CI. All participants received test batteries with both objective hearing tests (speech perception in noise and sound localisation) and subjective questionnaires.

Results 16 participants opted for BAHS-, 13 for CROS- and 30 for CI-treatment. The greater the subjective impairment caused by SSD, the more likely patients were to opt for surgical treatment (BAHS or CI). The best results in terms of speech perception in noise (especially when sound reaches the deaf ear and noise the hearing ear), sound localization, and subjective results were achieved with CI.

Conclusion The best results regarding the therapy of SSD are achieved with a CI, followed by BAHS. This was evident both in objective tests and in the subjective questionnaires. Nevertheless, an individual decision is required in each case as to which SSD therapy option is best for the patient. Above all, the patient's subjective impairment and expectations should be included in the decision-making process.
\end{abstract}

Keywords Single-sided deafness $\cdot$ Cochlear implant $\cdot$ Bone anchored hearing system · Contralateral routing of signals hearing aids hearing aid $\cdot$ SSQ

\section{Introduction}

People who suffer from single-sided deafness (SSD) have difficulty understanding speech, especially when the sound comes to the deaf ear or in noise, as well as in the localization of sounds [1]. Patients with SSD should be advised in detail about the various options for hearing rehabilitation. In particular, the limitations as well as the advantages and disadvantages of the different devices should be discussed. In addition to the avoidance of technical support with the

Till F. Jakob

till.jakob@uniklinik-freiburg.de

1 Department of Oto-Rhino-Laryngology, Medical Center - University of Freiburg, Faculty of Medicine, University of Freiburg, Germany, Killianstraße 5, 79106 Freiburg, Germany aim of developing compensatory mechanisms (especially for sound localisation when hearing loss occurred in childhood; [2]), there are three different options for rehabilitation of the deaf ear based on a device. Over the last few years, the treatment with best results for unilateral deaf patients has been cochlear implant (CI; [3-5]), whenever possible and desired by the patient. Alternative treatment options for SSD are contralateral routing of signals hearing aids (CROS) [6] or bone-anchored hearing systems (BAHS) [7]. The BAHS can be worn on a headband, transcutaneously or percutaneously. Binaural hearing is possible with neither CROS nor BAHS and no beneficial effect regarding speech perception in noise and sound localisation is shown in a review [8] although one study showed an improvement in speech-in-noise performance for SSD [9]. Advantages of a CI are the restoration of binaural hearing and the associated better speech perception in noise and better sound localisation [4]. Aim 
of this study was to investigate benefits of different hearing rehabilitation options in SSD focusing on: Which device (BAHS, CROS, or CI) provides the best results in objective hearing tests after 12 months of using the selected device? Based on questionnaires, what is the subjective benefit of the individual devices? What are the factors that may influence the decision for a particular hearing device? Are there differences between immediate testing and in long-term use after 12 months?

\section{Materials and methods}

\section{Study design and participants}

In this prospective study, 89 adult (age $>18$ years) patients with SSD were included. The study was conducted in accordance with the recommendations of the Ethics Committee of the University of Freiburg, Germany (protocol numbers 175/08 and 69/09) and in accordance with the Declaration of Helsinki (2013). The audiological classification of SSD was: untreated hearing ability of the poorer-hearing ear with a mean bone-conduction audiometric threshold for the frequencies 500, 1000, 2000, $4000 \mathrm{~Hz}$ (bone-conduction four-frequency pure-tone average, $\mathrm{BC} 4 \mathrm{PTA}$ ) greater than or equal to $70 \mathrm{~dB}$ hearing level (HL) and an air-conduction 4PTA of less than or equal to $30 \mathrm{~dB}$ HL for the betterhearing ear [10]. The first part of the study is a prospective descriptive cohort study with a randomised 3-week test period of BAHS and CROS devices. The second part of the study is the 12-month follow-up after choosing the treatment option (BAHS, CROS or CI).

After signed informed consent was obtained, an audiological assessment was performed in all participants at baseline including the following measures: pure-tone hearing thresholds, speech intelligibility for Freiburg monosyllables and numbers, tympanograms, and auditory brainstem responses (ABR). In participants who decided for a CI, transtympanal electrocochleography or promontorial testing, temporal bone $\mathrm{CT}$ and MRI were performed. After the test periods of randomised $2 \times 3$ weeks ( 3 -week BAHS-testing on head band and 3-week CROS-testing) the participants were divided into three groups: Group A: participants who decided on a BAHS, group B: participants who decided on a CROS hearing aid, and group C: participants who decided on a CI. The participants of groups $\mathrm{A}$ and $\mathrm{C}$ were then implanted (usually BAHS under local anaesthesia and CI under general anaesthesia). The outcomes of some patients were discussed in previous publications $[4,5]$.

\section{Study Plan}

All study participants completed a 3-week test phase with CROS and BAHS. Which device was tested first was determined randomly. After each of the test phases, the participants filled out questionnaires in which their subjective experiences were recorded. Hearing tests were also performed to determine the objective hearing ability. In addition, all participants who were in principle eligible for a CI were informed about the possibility of CI treatment. After the test phase, the participants could decide whether they wanted to continue participating in the study and what kind of hearing rehabilitation they wanted. Therefore, the number of participants in each group is different. The tests were repeated after 12 months for the participants in groups $\mathrm{A}$ and B. For group C participants, the tests were repeated after 6 and after 12 months. Testing after 6 months was performed routinely as part of rehabilitation (Fig. 1).

\section{Test battery}

Speech in noise testing: Speech intelligibility in noise was assessed using the Hochmair-Schulz-Moser (HSM) sentence test [11]. Speech and background noise were presented at $65 \mathrm{~dB}$ SPL, i.e., with a fixed signal-to-noise ratio (SNR) of $0 \mathrm{~dB}$. Three speech $(\mathrm{S})$ in noise $(\mathrm{N})$ presentation conditions were examined: S0N0, SnhNssd and SssdNnh (nh, normal hearing side; ssd, single-sided deafness side). In presentation condition SON0, both speech and noise were presented from the front at an angle of $0^{\circ}$. In the presentation condition SnhNssd, speech was presented from the normal-hearing side and background noise from the deaf side at an angle of $+45^{\circ}$ and $-45^{\circ}$, and vice versa in the presentation condition SssdNnh (speech from the deaf side and background noise from the normal-hearing side each at an angle of $45^{\circ}$; setup according to Arndt et al. [4, 12]). The tests were performed at the beginning of the study without any hearing aid, after a 3-week test trial with BAHS and after a 3-week test trial with CROS, and after 12 months with the supplied device (in case of CI also after 6 months; see Fig. 1).

Localisation: Localisation abilities were tested in a sound-isolated room with seven loudspeakers positioned in a frontal semicircle $2 \mathrm{~m}$ in diameter with a distance of $30^{\circ}$ in the horizontal plane around the participant at head level. Sentences of the Oldenburg sentence test (OLSA) [13] were used as stimuli for the assessment of localisation ability. In each localisation test, 70 sentences (10 per loudspeaker) were presented at sound levels of 59, 62, 65, 68 and $71 \mathrm{~dB}$ SPL and a mean sound level of $65 \mathrm{~dB}$ SPL, in random sequence from one of the 7 loudspeakers. For each participant and each condition, the localisation ability was measured as the angle error in degrees, that is, as the mean 


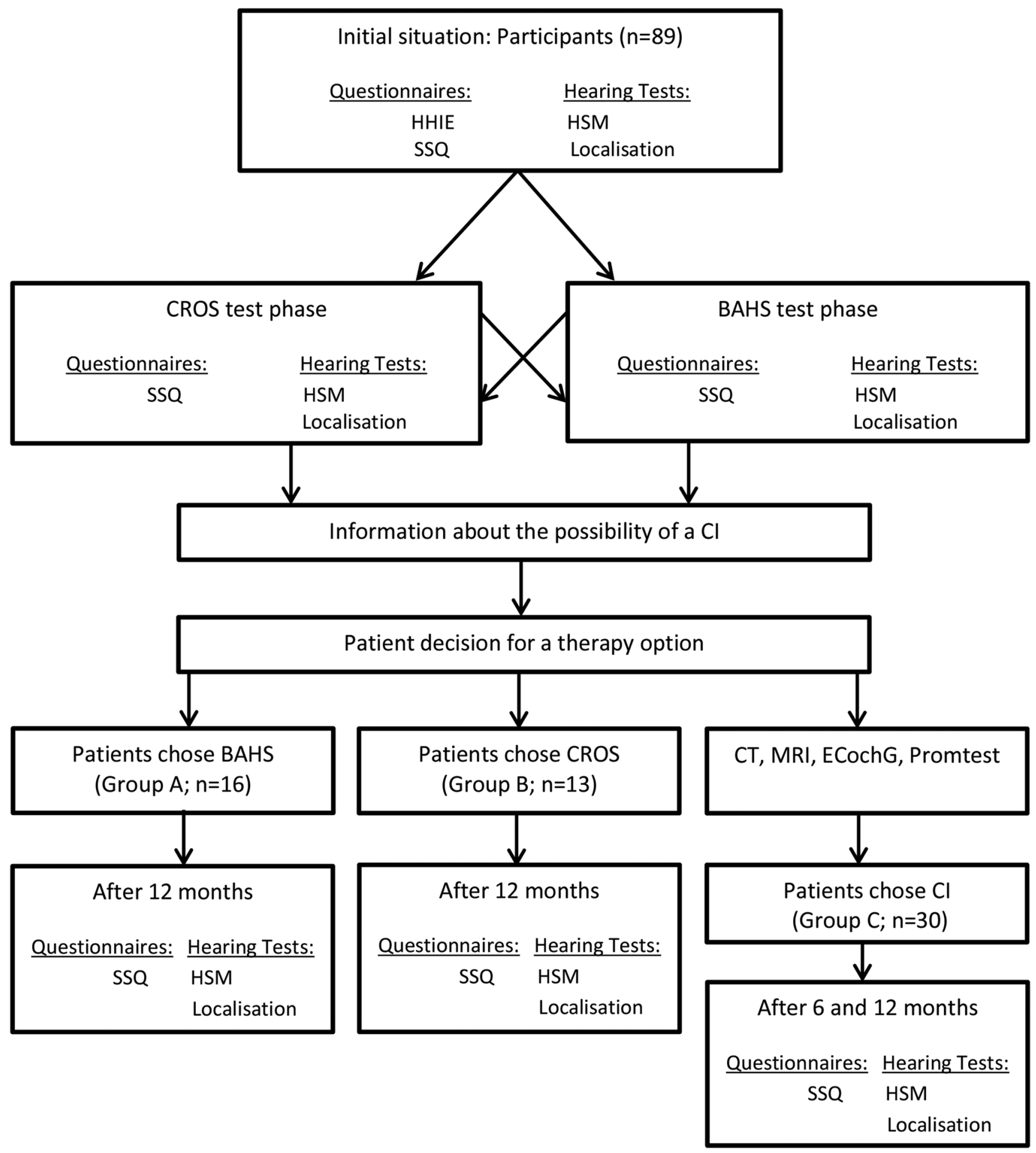

Fig. 1 Flowchart of study design: Of the initial 89 participants, 59 participants decided to continue the study. 16 participants underwent BAHS surgery, 13 chose CROS hearing aids and 30 participants underwent CI surgery. The times of the hearing tests and questionnaires are shown in the flowchart. BAHS bone-anchored hearing system, $C I$ cochlear implant, CROS contralateral routing of signals,
ECoch $G$ Electrocochleography, HHIE Hearing Handicap Inventory for Adults, HSM Hochmair-Schulz-Moser sentence test, IOI-HA International Outcome Inventory for Hearing Aids, OLSA Oldenburg sentence test, Promtest promontorial testing, SSQ Speech, Spatial and Qualities of Hearing Scale 
difference in angle between the presentation loudspeaker and the loudspeaker identified by the patient (setup according to Arndt et al. [4, 12]).

\section{Questionnaires}

Hearing Handicap Inventory for Adults (HHIA): This tenitem questionnaire records the subjectively perceived limitation on a social and emotional level that the patient brings into the study due to his single-sided deafness [14]. Since the questionnaire was intended to capture the initial situation, it was only applied at the beginning of the study. The degree of subjective impairment is divided into three groups: no hearing handicap ( $0-8$ points), mild-to-moderate hearing handicap (10-24 points) and significant hearing handicap (26-40 points).

Speech, Spatial and Qualities of Hearing Scale (SSQ): This 3 -section questionnaire assesses speech understanding, spatial hearing, and hearing quality with a scoring system of 0 to 10 for each item. Unable to hear is represented by 0 and 10 means hears perfectly [15].

\section{Data Analysis}

Statistical analysis was carried out in GNU R (R Core Team, 2014) and illustrated by Box-Whisker plots. The Shapiro-Wilk test was used to check the data for normal distribution. The Kruskal-Wallis test was performed for comparison of the three groups (A-C) in SSQ, localisation ability and speech recognition in noise. For post hoc analyses, pairwise comparison with the Wilcoxon-rank tests using Bonferroni correction was applied. A level of significance of 0.05 was applied in all analyses.

\section{Results}

\section{Participants}

In this study, 89 adult participants were included with an average age of 55.8 years and an average period of singlesided deafness of 10.7 years ( 1 month to 51.1 years; average age and period of SSD for each group, see Table 1). There was no significant difference in age between the groups $(p=0.359)$. However, we found a difference between the groups in the duration of deafness $(p=0.007)$. The duration of deafness was shorter for group $C$ than in the other groups (Group A vs. Group C: $p=0.004$; Group B vs. Group C: $p=0.029$; no treatment vs. Group C: $p=0.008$ ). After the two trials, 16 participants chose BAHS (Group A; implants: 12 Cochlear $^{\mathrm{TM}}$ Baha BP100®, 3 Oticon Medical ${ }^{\mathrm{TM}}$ Ponto Power ${ }^{\circledR}, 1$ Cochlear ${ }^{\mathrm{TM}}$ Baha Intenso $\left.{ }^{\circledR}\right), 13$ participants chose CROS (Group B; Phonak Una M), and 30 participants chose CI (Group C; implants: 17 Cochlear ${ }^{\mathrm{TM}}$ Nucleus ${ }^{\circledR}$ CI512, 12 Cochlear ${ }^{\mathrm{TM}}$ Nucleus ${ }^{\circledR}$ CI24RE, 1 Cochlear ${ }^{\mathrm{TM}}$ Nucleus ${ }^{\circledR}$ CI422). 20 participants decided against treatment and 10 participants stopped the study. The main reason for SSD was sudden hearing loss $(n=29)$, followed by unclear reason of SSD since childhood $(n=13)$. For further aetiologies, see Table 1.

Table 1 Demographic characteristics per group

\begin{tabular}{|c|c|c|c|c|}
\hline Group & No treatment & BAHS (group A) & CROS (group B) & CI (group C) \\
\hline Number & 20 & 16 & 13 & 30 \\
\hline Gender & $\begin{array}{l}\mathrm{f}: n=13 \\
\mathrm{~m}: n=7\end{array}$ & $\begin{array}{l}\text { f: } n=6 \\
\mathrm{~m}: n=10\end{array}$ & $\begin{array}{l}\mathrm{f}: n=5 \\
\mathrm{~m}: n=8\end{array}$ & $\begin{array}{l}\text { f: } n=15 \\
\text { m: } n=15\end{array}$ \\
\hline Age $($ mean $\pm S D)$ & $55.0 \pm 13.1$ years & $60.8 \pm 13.9$ years & $56.8 \pm 15.4$ years & $53.4 \pm 12.3$ years \\
\hline Deafness duration (mean $\pm \mathrm{SD}$ ) & $187.6 \pm 197.3$ months & $158.3 \pm 186.1$ months & $221.8 \pm 227.6$ months & $34.7 \pm 45.9$ months \\
\hline 4PTA better ear $($ mean \pm SD) & $13.5 \pm 8.4 \mathrm{~dB}$ & $17.8 \pm 12.6 \mathrm{~dB}$ & $16.6 \pm 10.8 \mathrm{~dB}$ & $15.6 \pm 14.4 \mathrm{~dB}$ \\
\hline PTA poorer ear $($ mean \pm SD) & $100.8 \pm 20.8 \mathrm{~dB}$ & $99.6 \pm 21.6 \mathrm{~dB}$ & $116.1 \pm 21.7 \mathrm{~dB}$ & $104.7 \pm 22.3 \mathrm{~dB}$ \\
\hline Aetiology: Sudden idiopathic hearing loss & 8 & 4 & 4 & 13 \\
\hline Unknown since childhood & 5 & 3 & 5 & 0 \\
\hline Vestibular schwannoma & 1 & 4 & 3 & 0 \\
\hline Labyrinthitis & 0 & 0 & 1 & 5 \\
\hline Menière's disease & 2 & 0 & 0 & 1 \\
\hline After ear surgery & 1 & 2 & 0 & 3 \\
\hline Otosclerosis & 0 & 1 & 0 & 3 \\
\hline Meningitis & 2 & 0 & 0 & 1 \\
\hline Temporal bone fracture & 1 & 0 & 0 & 1 \\
\hline Mumps & 0 & 1 & 0 & 1 \\
\hline Otitis media & 0 & 1 & 0 & 1 \\
\hline Cogan-1-Syndrome & 0 & 0 & 0 & 1 \\
\hline
\end{tabular}




\section{Results of HHIA}

Of the participants, 5.5\% (3 of 55) felt no hearing handicap (0-8 points), $40.0 \%$ (22 of 55) felt a mild-to-moderate hearing handicap (10-24 points) and $54.5 \%$ (30 of 55) a significant hearing handicap (26-40 points). Four patients did not return the questionnaire. Group A rated their hearing handicap on average with 26.8 points (SD 9.6), Group B with 18 points (SD 8.9) and Group C with 28.2 points (SD 9.9). BAHS (9 of 15) and CI (19 of 29) were mostly chosen by participants with severe impairment and CROS ( 8 of 11) by participants with mild-to-moderate impairment.

\section{Test batteries}

\section{Speech in noise (HSM sentence test)}

Results of HSM sentence test at different time points, different treatment options, and different presentation configurations are shown in Fig. 2A-C.

Group A: After the 3-week test period with a headband, no significant improvements for BAHS were found in all test configurations (S0N0: $p=0.4351$, SssdNnh: $p=0.06921$, SnhNssd: $p=1$; Fig. $2 \mathrm{a}-\mathrm{c}$ ). Although not significant, there is a trend in the SssdNnh situation (median untreated $=0 \%$; median BAHS $=5.66 \%$; Fig. 2b). After CROS testing, the SssdNnh situation showed a significant improvement compared to the untreated situation $(p=0.003$; median unaided $=0 \%$; median CROS $=27.83 \%$; Fig. $2 b$ ). In the SnhNssd situation, however, a significant deterioration in speech understanding was observed when using a CROS $(\mathrm{p}=0.010$; median unaided $=97 \%$; median $\mathrm{CROS}=81 \%$; Fig. 2c). After implantation of the BAHS and testing after 12 months, there was a significant improvement in the SssdNnh configuration ( $p=0.008$; median unaided $=0 \%$, median BAHS $12 \mathrm{~m}=40.59 \%$; Fig. 2b).

Group B: After the 3-week test period with the CROS system, no significant improvements were found in all test configurations (S0N0: $p=0.570$; SssdNnh: $p=0.151$; SnhNssd: $p=0.101$; Fig. 2a-c). No differences were found after BAHS testing, either (S0N0: $p=0.570$; SssdNnh: $p=1$; SnhNssd: $p=1$; Fig. 2a-c). After 12 months there was also no improvement with CROS. Although not significant, CROS seems to be associated with poorer speech comprehension compared to the unaided situation in the SnhNssd configuration (median unaided $=98.58 \%$; median CROS $=79.25 \%, p=0.101$; median CROS $12 \mathrm{~m}=64.62 \%$, $p=0.603)$.

Group C: BAHS testing, CROS testing and CI after 12 months showed no differences in the S0N0 $(p=0.088)$ configuration compared to the untreated situation. In the SssdNnh configuration, all 3 devices significantly improved speech understanding (unaided vs. BAHA: $p=0.005$; unaided vs. CROS: $p<0.001$; unaided vs. CI 12 months: $p<0.001)$. In the SnhNssd configuration, the CROS resulted in a significant decrease in speech understanding compared to the unaided situation (unaided vs. CROS: $p=0.001$ ).

Comparing the 12-month results between the three groups showed significantly better speech perception with $\mathrm{CI}$ in the SON0 configuration compared to the CROS group $(p=0.017)$ and better speech perception in the SnhNssd configuration compared to BAHS group $(p=0.002)$ and CROS group $(p<0.001)$.

\section{Localisation}

Localisation errors of all three groups are shown in Fig. 3. Localisation error is significantly reduced in the CI group after 12 months by $10.93^{\circ}$ (median unaided $26.36^{\circ}$, median CI $12 \mathrm{~m}=15.43^{\circ} ; p<0.001$ ) compared to the unaided conditions. No differences were found in Groups A and B. Comparing the 12-month results there was a significant difference between Group B and C ( $p=0.008$; A vs. B: $p=0.095$; A vs. C: $p=0.151$ ).

\section{SSQ}

Speech understanding: In the speech section there is a significant improvement between the unaided situation (median 3.75) and group A test phase (median $6.61 ; p=0.024$ ) and after 12 months (median 6.39; $p=0.037$ ). No difference was found between the unaided situation (median 5.07) and group B after the CROS test phase (median $6.42 ; p=0.505$ ) and after 12 months (median 6.94; $p=0.236$ ). A significant difference was found between the unaided situation (median 3.05 ) and group $C$ after 6 months (median 5.54; $p<0.0001$ ) and after 12 months (median 5.92; $\mathrm{p}<0.0001$ ). In all groups no differences were found between the test phase and after 12 months. No differences were found between the three groups after 12 months ( $p=0.392$; Fig. 4A).

Spatial hearing:

In the spatial section of group A, differences were found between the unaided situation (median 3.5) and after the test phase (median $6.48 ; p=0.008$ ) and, although not significant, between the unaided situation and after 12 months (median 6.0; $p=0.057$ ). Also in group $\mathrm{B}$, differences were found between the unaided situation (median 2.21) and after the test phase (median 4.76; $p=0.015$ ) but not between the unaided situation and after 12 months (median 5.65; $p=0.236$ ). In group $\mathrm{C}$, differences were found compared to the unaided situation (median 2.26) after 6 (median 5.18; $p<0.001$ ) and after 12 months (median 5.83; $p<0.001$ ). In all groups no differences were found between the early test phase and after 12 months. No differences were found between the three groups (Fig. 4B). 
Fig. 2 Box-Whisker plots of Hochmair-Schulz-Moser (HSM) sentence test: a SONO (S: speech; N: noise) presentation setup with speech and noise from the front. Group A: participants who chose BAHS; group B: participants who chose CROS; group C: participants who chose CI. b SssdNnh (ssd: single-sided deafness; nh: normal hearing) presentation setup with speech from the unilateral deaf side, noise from the normal hearing side. c SnhNssd presentation setup with speech from the normal hearing side, noise from the unilateral deaf side. $B A H S$ bone-anchored hearing system, $C I$ cochlear implant, CROS contralateral routing of signals

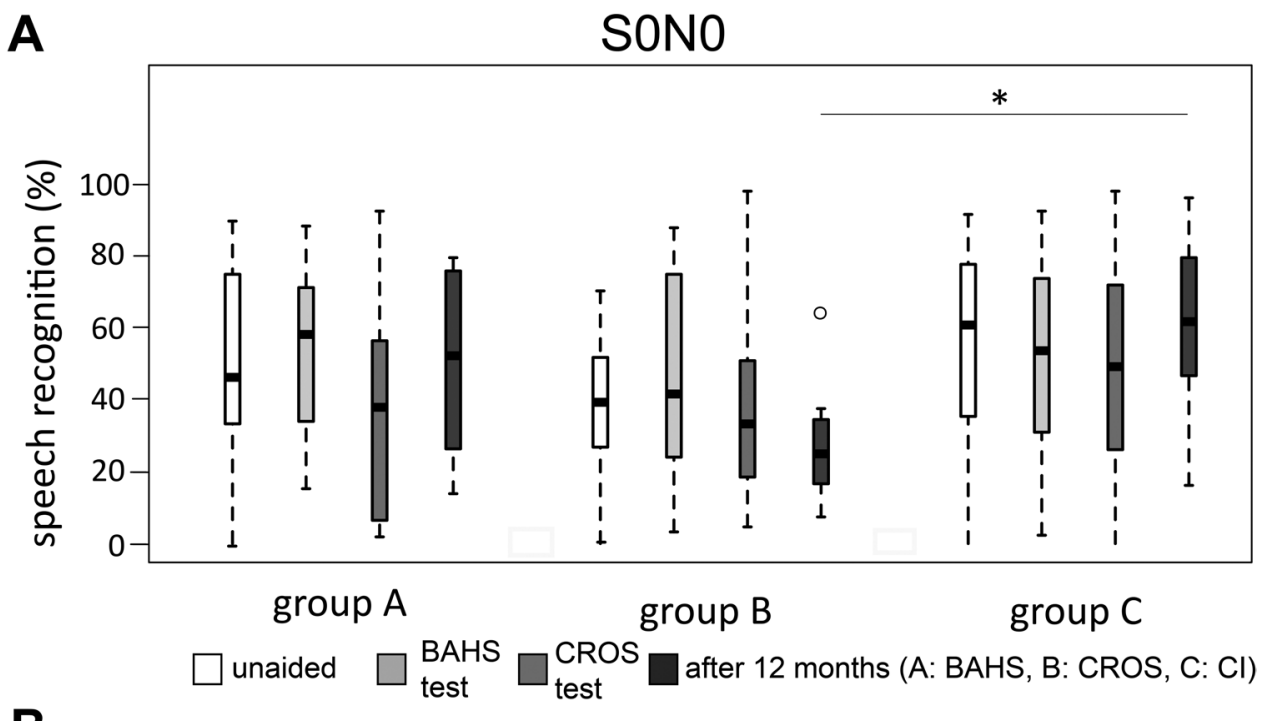

B

SssdNnh

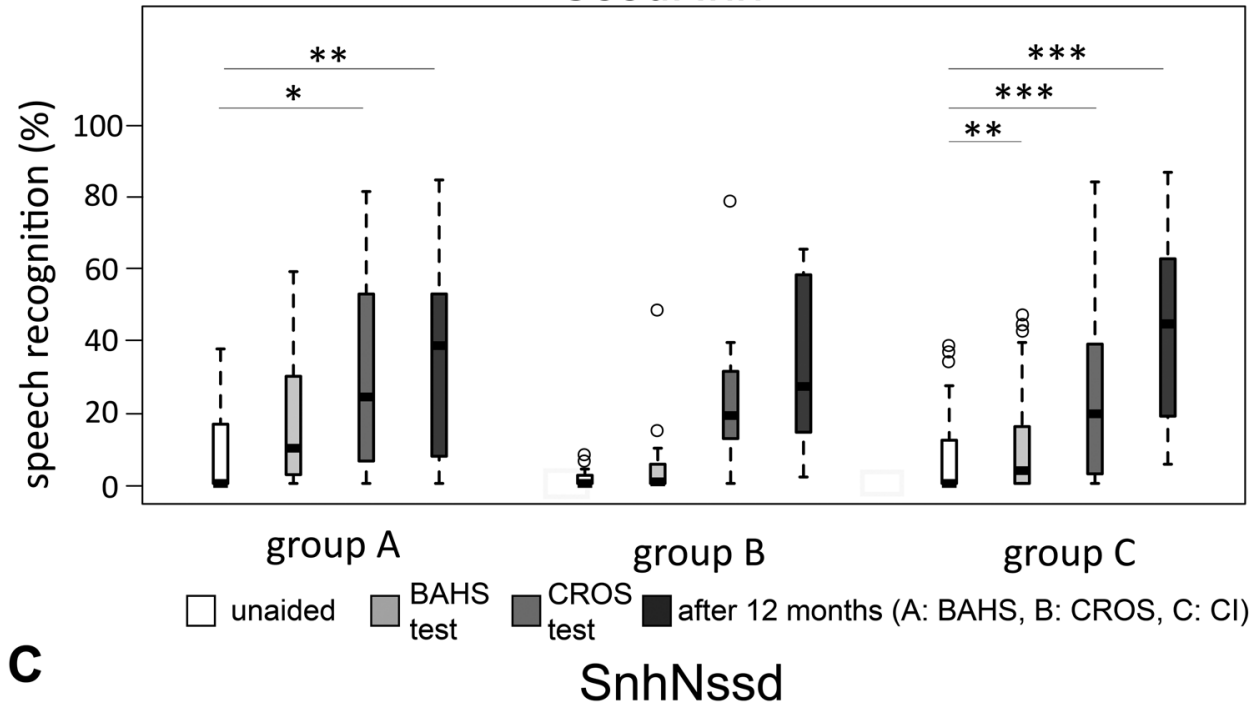


Fig. 3 Box-Whisker plots of localisation error: group A: participants who chose BAHS; group B: participants who chose CROS; group C: participants who chose CI. BAHS boneanchored hearing system, $C I$ cochlear implant, $C R O S$ contralateral routing of signals

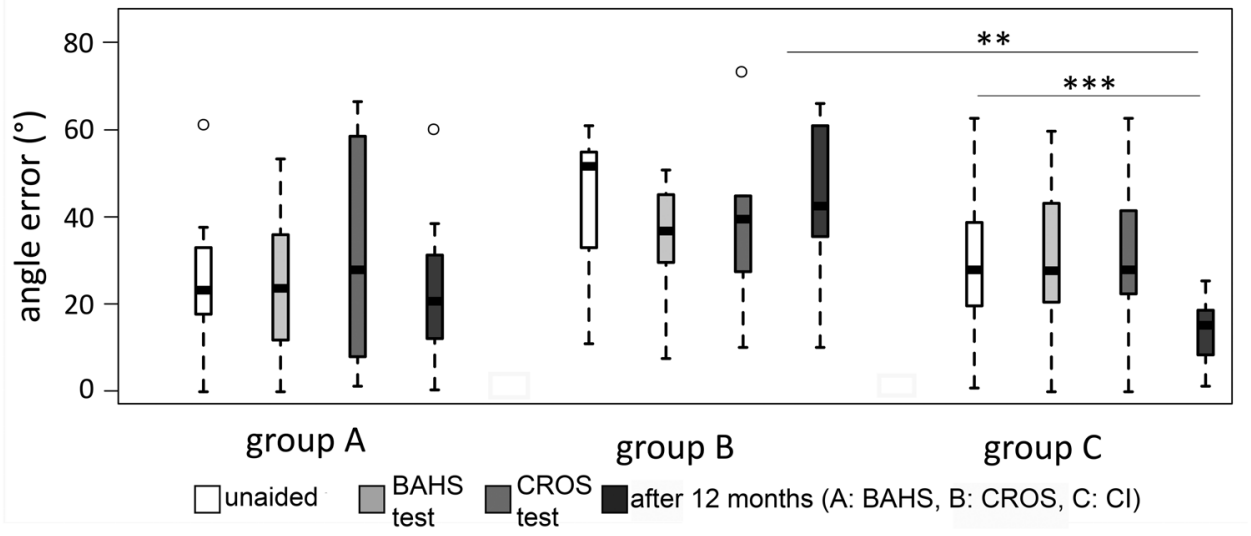

Hearing quality: In the quality section a difference was only found between group B (8.51) and group C (3.36) after 12 months ( $p=0.036$; Fig 4 C).

\section{Discussion}

Based on hearing tests and questionnaires, we compare in this study the results of three different treatment options for SSD, namely BAHS, CROS, and CI. Our results show an improvement in speech comprehension in the situation, where speech comes to the deaf ear and noise to the hearing ear for BAHS and CI. However, there is a negative effect using a CROS in the situation, where speech is applied to the hearing ear and noise to the deaf ear. The localisation of sounds can only be improved by a CI. Subjectively, there is a significant improvement after 12 months in speech understanding and spatial hearing in CI and in speech understanding in BAHS.

In terms of age, the groups were homogeneous, whereas there were differences in the duration of deafness. The shorter duration of deafness in the CI group can be explained by the fact that a CI is not a treatment option in cases of unknown deafness since childhood. In addition, in the case of labyrinthitis, which requires rapid CI implantation due to the risk of cochlear ossification, most patients chose a CI (5 vs. 1 in all other groups combined). In contrast to our 2011 study [4], we included not only patients in whom CROS and BAHS were not successful in the study. Since the patients in the recent study had the choice of which device to use, the group sizes are different. It is noticeable that patients with severe subjective limitations (high score in HHIA questionnaire) tended to opt for surgical therapy like BAHS and CI, whereas patients with less severe limitations tended to opt for a CROS hearing aid. This can be explained by the fact that patients with less subjective impairment from SSD do not want to take the effort and risk of surgery. In addition to the advantage that no surgery is required for a CROS hearing aid, it can also be easily removed and there is no implant or screw in the body. However, of the 13 patients who opted for CROS, only 6 patients came back for followup after 12 months. In the CI group all 30 and in the BAHS group all 16 could be re-tested after 12 months. This leads to the assumption that patients with a subjectively low hearing handicap who choose CROS are more likely to be non-users.

Numerous studies have shown improved speech perception and sound localization with a CI in SSD compared to untreated situations $[3,16]$ or to CROS and BAHS $[4$, 17]. However, these studies did not look at the results after 1 year. Also, the BAHS were worn with a headband and had not been implanted. The hearing results achieved with a headband are worse than with semi-implantable devices [18]. In the BAHS group, the SssdNnh configuration showed an improvement after 1 year with the implanted BAHS that seems to be better than after 3-week testing with a headband. In this configuration, speech comprehension of HSM sentences improved from $0 \%$ (unaided) and $6 \%$ after test trial with a headband to $40 \%$ with a percutaneous implanted device after 12 months.Similar results are found in a study, where speech perception with an anchored BAHS is found to be $14-20 \%$ better than with a headband [19]. Compared to other studies [20], no significant negative effect of the BAHS is found in the NssdSnh configuration. The CI does not negatively influence the situation, either. However, this negative effect is seen with a CROS in a reduction of speech comprehension from $99 \%$ unaided to $79 \%$ after the test phase and further to $65 \%$ (both not significant) after 1 year. In the situation, where noise affects the better ear, a meta-analysis was able to show that a negative effect occurs especially in CROS, followed by BAHS [21]. Comparing the HSM results of the three devices after 1 year, the CI group seems to have the best speech comprehension in all three tested configurations (HSM 12 m SON0: BAHS: 53\%; CROS 29\%, CI: 63\%; SssdNnh: BAHS $=41 \%, \mathrm{CROS}=18 \%, \mathrm{CI}=47 \%$; SnhNssd: BAHS $=84 \%$, CROS $=65 \%, C I=99$ ). 
Fig. 4 Box-Whisker plots of Speech, Spatial and Qualities of Hearing Scale (SSQ): group A: participants who chose BAHS; group B: participants who chose CROS; group C: participants who chose CI. a Speech understanding; b Spatial hearing; c Hearing quality: For a-c Unable to hear is represented by 0 and 10 means hears perfectly. BAHS bone-anchored hearing system, $C I$ cochlear implant, $C R O S$ contralateral routing of signals
A Speech

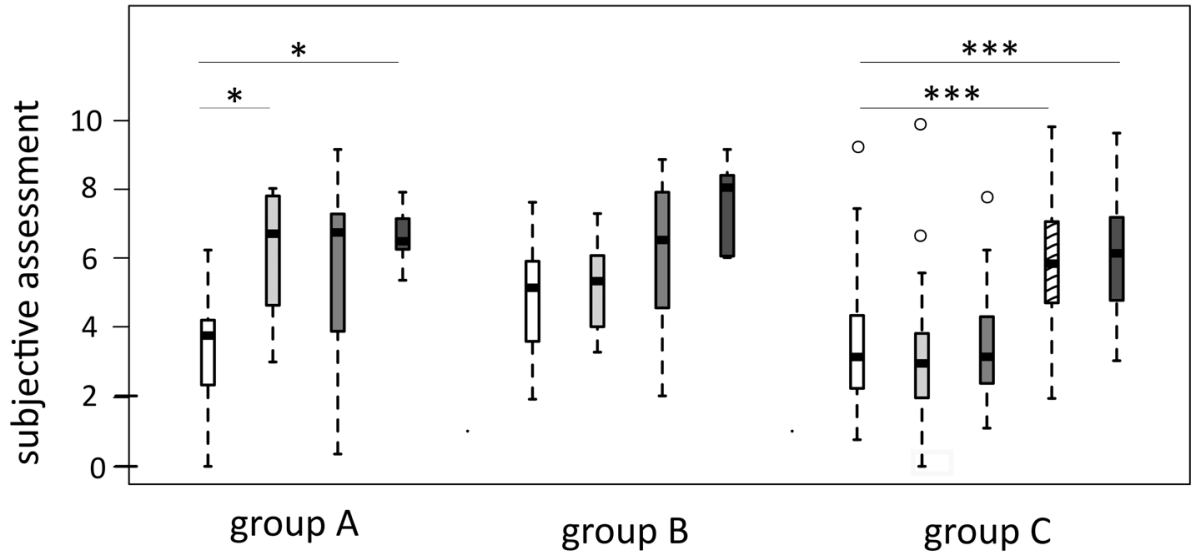

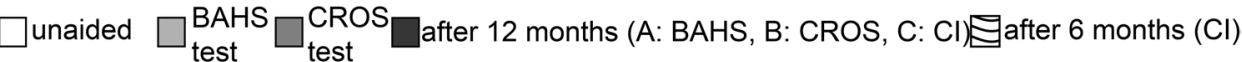

B Spatial

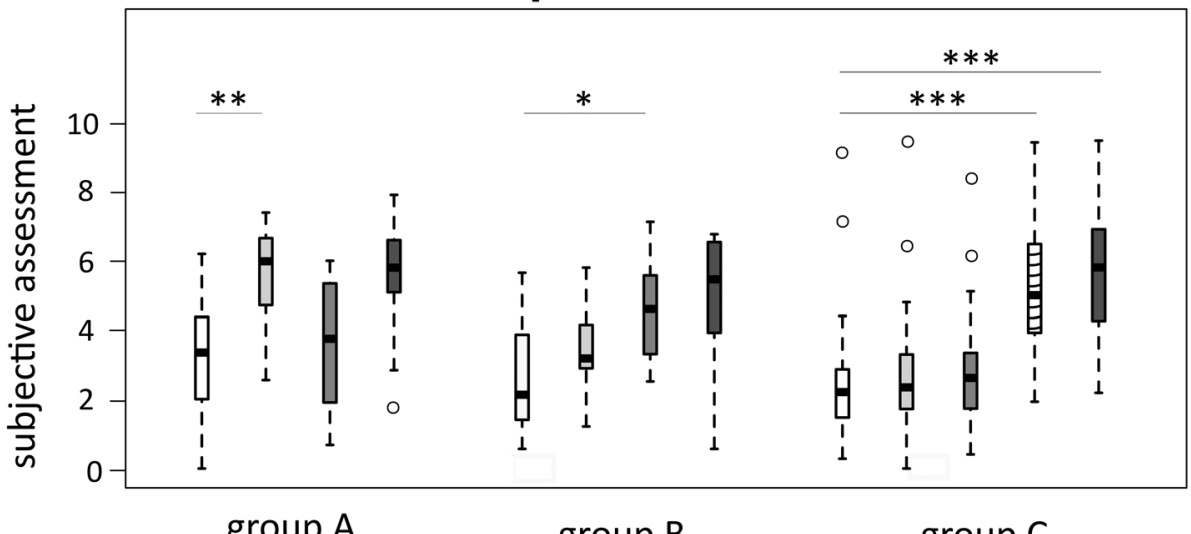

$\square$ unaided $\square$ BAHS $\square$ CROS $\square$ after 12 months (A: BAHS, B: CROS, C: CI) $\bigoplus$ after 6 months (Cl)
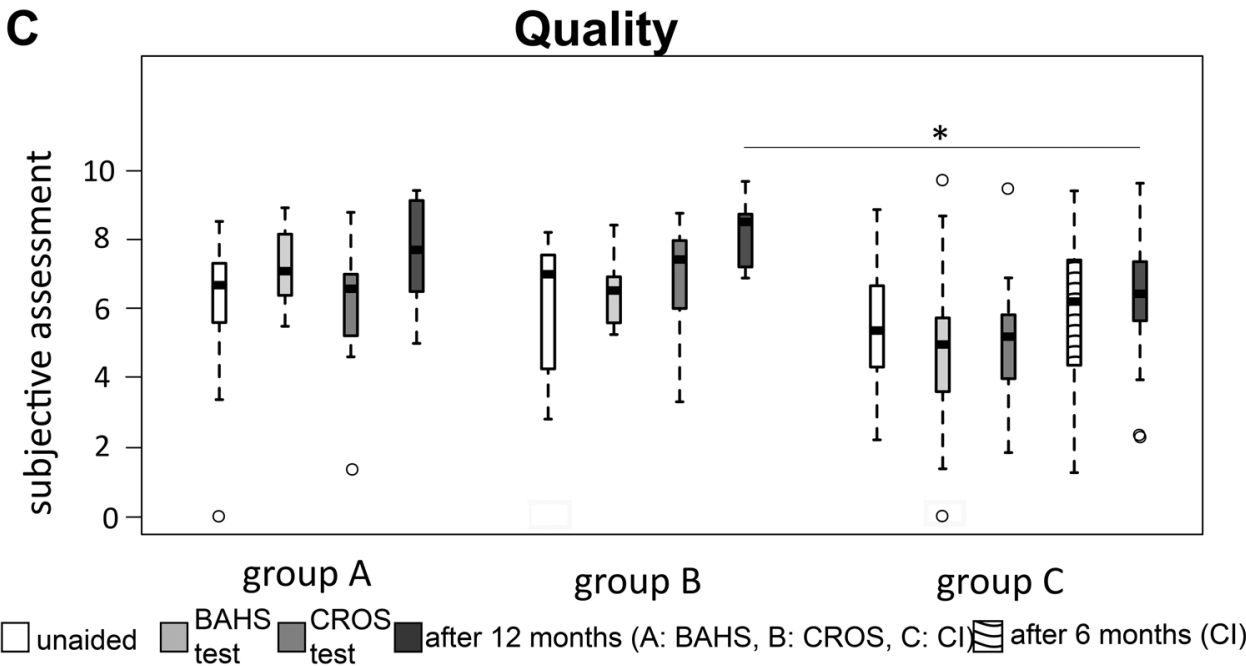

Sound localisation was best with CI, with a reduction of the localisation error of $10.93^{\circ}$ compared to the unaided situation. The improvement to localise sound by a CI has been shown in numerous studies (for reviews see: [21-23]). We found no benefits regarding sound localisation for the BAHS or CROS group, this is also shown in other studies regarding sound localization (for reviews see: [8, 24]). Other studies 
even showed significant deficits in localisation performance for BAHS [25] and for CROS [26].

Based on the SSQ questionnaire, speech understanding is improved by BAHS and CI, but not by CROS. Spatial hearing improves subjectively after 12 months only with CI. Hearing quality did not improve statistically with the three devices. Hearing-related quality of life can be measured by the SSQ scale and by the abbreviated profile of hearing aid benefit (APHAB). The latter measures ease of communication, background noise, reverberation and aversion to loud sounds. A meta-analysis found significant benefits for BAHS for all subscales of the APHAB except aversion to loud sounds, for CROS significant benefits were only found for background noise and reverberation. A meta-analysis for SSQ did not identify significant effects of BAHS or CROS compared to the unaided situation, but significant decreases in listening difficulty with CI was found on all subscales [21]. In our previous study, we showed an advantage of the CI in the SSQ over BAHS and CROS, but that was one group that tested all 3 devices and only participants were enrolled whose conventional therapy with CROS and BAHS had not been successful [4].

\section{Limitations}

A disadvantage of the study is the greater ceiling effect when using the HSM with fixed noise and speech level instead of an adaptive procedure. However, since the same test was performed in all three groups, the groups are comparable. A further disadvantage is the different size of the groups, which cannot be avoided, because the patient is allowed to choose the device and is not randomly assigned to a group. Therefore, the groups are not randomized, which results in a further inevitable bias.

\section{Conclusion}

In summary, the best results regarding therapy of SSD are possible with a CI, followed by BAHS. This was evident both in objective tests and in the subjective questionnaires. Nevertheless, an individual decision is required in each case as to which therapy option for SSD is best for the patient. Above all, the patient's subjective impairment and expectations should be included in the decision-making process.

Acknowledgements The authors thank Julian Kubens for collecting the data and Ms. Joanne Eysell for providing language assistance.

Funding Open Access funding enabled and organized by Projekt DEAL.

\section{Compliance with ethical standards}

Informed consent All participants signed informed consent statements before inclusion in the study. Bone-anchored hearing system, contralateral routing of signals hearing aid or cochlear implant: what is best in single-sided deafness?

Open Access This article is licensed under a Creative Commons Attribution 4.0 International License, which permits use, sharing, adaptation, distribution and reproduction in any medium or format, as long as you give appropriate credit to the original author(s) and the source, provide a link to the Creative Commons licence, and indicate if changes were made. The images or other third party material in this article are included in the article's Creative Commons licence, unless indicated otherwise in a credit line to the material. If material is not included in the article's Creative Commons licence and your intended use is not permitted by statutory regulation or exceeds the permitted use, you will need to obtain permission directly from the copyright holder. To view a copy of this licence, visit http://creativecommons.org/licenses/by/4.0/.

\section{References}

1. Giolas TG, Wark DJ (1967) Communication problems associated with unilateral hearing loss. J Speech Hear Disord 32(4):336-343. https://doi.org/10.1044/jshd.3204.336

2. Firszt JB, Reeder RM, Holden LK (2017) Unilateral hearing loss: understanding speech recognition and localization variability implications for cochlear implant candidacy. Ear Hear 38(2):159173. https://doi.org/10.1097/AUD.0000000000000380

3. Vermeire K, van de Heyning P (2009) Binaural hearing after cochlear implantation in subjects with unilateral sensorineural deafness and tinnitus. Audiol Neurootol 14(3):163-171. https://doi. org/10.1159/000171478

4. Arndt S, Aschendorff A, Laszig R et al (2011) Comparison of pseudobinaural hearing to real binaural hearing rehabilitation after cochlear implantation in patients with unilateral deafness and tinnitus. Otol Neurotol 32(1):39-47. https://doi. org/10.1097/MAO.0b013e3181fcf271

5. Arndt S, Laszig R, Aschendorff A et al (2011) Einseitige Taubheit und Cochlear-implant-Versorgung: Audiologische Diagnostik und Ergebnisse (Unilateral deafness and cochlear implantation: audiological diagnostic evaluation and outcomes). HNO 59(5):437-446. https://doi.org/10.1007/s00106-011-2318-8

6. Snapp H (2019) Nonsurgical management of single-sided deafness: contralateral routing of signal. J Neurol Surg B Skull Base 80(2):132-138. https://doi.org/10.1055/s-0039-1677687

7. Niparko JK, Cox KM, Lustig LR (2003) Comparison of the bone anchored hearing aid implantable hearing device with contralateral routing of offside signal amplification in the rehabilitation of unilateral deafness. Otol Neurotol 24(1):73-78. https://doi. org/10.1097/00129492-200301000-00015

8. Peters JPM, Smit AL, Stegeman I et al (2015) Review: Bone conduction devices and contralateral routing of sound systems in single-sided deafness. Laryngoscope 125(1):218-226. https ://doi.org/10.1002/lary.24865

9. Snapp HA, Holt FD, Liu X et al (2017) Comparison of speechin-noise and localization benefits in unilateral hearing loss subjects using contralateral routing of signal hearing aids or bone-anchored implants. Otol Neurotol 38(1):11-18. https://doi. org/10.1097/MAO.0000000000001269

10. van de Heyning P, Távora-Vieira D, Mertens G et al (2016) Towards a unified testing framework for single-sided deafness 
studies: a consensus paper. Audiol Neurootol 21(6):391-398. https://doi.org/10.1159/000455058

11. Hochmair-Desoyer I, Schulz E, Moser L et al (1997) The HSM sentence test as a tool for evaluating the speech understanding in noise of cochlear implant users. Am J Otol 18(6 Suppl):S83

12. Arndt S, Laszig R, Aschendorff A et al (2017) CochleaImplantat-Versorgung von Patienten mit einseitiger Taubheit oder asymmetrischem Hörverlust (Cochlear implant treatment of patients with single-sided deafness or asymmetric hearing loss). HNO 65(Suppl 2):98-108. https://doi.org/10.1007/s0010 6-016-0297-5

13. Wagener K, Kühnel V, Kollmeier B (1999) Development and evaluation of a German sentence test I: Design of the Oldenburg sentence test. Zeitschrift Fur Audiologie 38:4-15

14. Newman CW, Weinstein BE, Jacobson GP et al (1991) Testretest reliability of the hearing handicap inventory for adults. Ear Hear 12(5):355-357. https://doi.org/10.1097/00003446-19911 0000-00009

15. Gatehouse S, Noble W (2004) The speech, spatial and qualities of hearing scale (SSQ). Int J Audiol 43(2):85-99. https://doi. org/10.1080/14992020400050014

16. Távora-Vieira D, de Ceulaer G, Govaerts PJ et al (2015) Cochlear implantation improves localization ability in patients with unilateral deafness. Ear Hear 36(3):e93-e98. https://doi.org/10.1097/ AUD.0000000000000130

17. Kurz A, Rak K, Hagen R et al (2020) Evaluating the decision for cochlear implantation in individuals with single-sided deafness (SSD) Implementing the SSD; Consensus Protocol Into Clinical Routine. Otol Neurotol. https://doi.org/10.1097/MAO.00000 00000002618

18. Rainsbury JW, Williams BA, Gulliver M et al (2015) Preoperative headband assessment for semi-implantable bone conduction hearing devices in conductive hearing loss: is it useful or misleading? Otol Neurotol 36(2):e58-62. https://doi.org/10.1097/MAO.00000 00000000695

19. Gründer I, Seidl RO, Ernst A et al (2008) Wertigkeit der BAHATestung für das postoperative Hörergebnis (Relative value of
BAHA testing for the postoperative audiological outcome). HNO 56(10):1020-1024. https://doi.org/10.1007/s00106-007-1608-7

20. Linstrom CJ, Silverman CA, Yu G-P (2009) Efficacy of the boneanchored hearing aid for single-sided deafness. Laryngoscope 119(4):713-720. https://doi.org/10.1002/lary.20164

21. Kitterick PT, Smith SN, Lucas L (2016) Hearing instruments for unilateral severe-to-profound sensorineural hearing loss in adults: a systematic review and meta-analysis. Ear Hear 37(5):495-507. https://doi.org/10.1097/AUD.0000000000000313

22. Vlastarakos PV, Nazos K, Tavoulari E-F et al (2014) Cochlear implantation for single-sided deafness: the outcomes. An evidence-based approach. Eur Arch Otorhinolaryngol 271(8):21192126. https://doi.org/10.1007/s00405-013-2746-Z

23. van Zon A, Peters JPM, Stegeman I et al (2015) Cochlear implantation for patients with single-sided deafness or asymmetrical hearing loss: a systematic review of the evidence. Otol Neurotol 36(2):209-219. https://doi.org/10.1097/MAO.000000000000068 1

24. Kim G, Ju HM, Lee SH et al (2017) Efficacy of bone-anchored hearing aids in single-sided deafness: a systematic review. OtolNeurotol 38(4):473-483. https://doi.org/10.1097/MAO.00000 00000001359

25. Grantham DW, Ashmead DH, Haynes DS et al (2012) Horizontal plane localization in single-sided deaf adults fitted with a boneanchored hearing aid (Baha). Ear Hear 33(5):595-603. https://doi. org/10.1097/AUD.0b013e3182503e5e

26. Lin L-M, Bowditch S, Anderson MJ et al (2006) Amplification in the rehabilitation of unilateral deafness: speech in noise and directional hearing effects with bone-anchored hearing and contralateral routing of signal amplification. Otol Neurotol 27(2):172-182. https://doi.org/10.1097/01.mao.0000196421.30275.73

Publisher's Note Springer Nature remains neutral with regard to jurisdictional claims in published maps and institutional affiliations. 